\section{(6) OPEN ACCESS}

\title{
Dual screen and confirm rapid test does not reduce overtreatment of syphilis in pregnant women living in a non-venereal treponematoses endemic region: a field evaluation among antenatal care attendees in Burkina Faso
}

\author{
Céline Langendorf, ${ }_{1}^{1}$ Céline Lastrucci, ${ }^{2}$ Isabelle Sanou-Bicaba, ${ }^{3}$ Kara Blackburn, ${ }_{1}^{2}$ \\ Marie-Hortense Koudika, ${ }^{2}$ Tania Crucitti $^{4}$
}

'Epicentre, Paris, France ${ }^{2}$ Médecins Sans Frontières (MSF), Paris, France ${ }^{3}$ Health family, Ministry of Health, Ouagadougou, Burkina Faso

${ }^{4}$ Department of Clinical Sciences, Institute of Tropical Medicine, Antwerp, Belgium

\section{Correspondence to}

Céline Langendorf, Epicentre, Paris 75011, France; celine. langendorf@epicentre.msf.org

Received 13 June 2018 Revised 31 October 2018 Accepted 25 November 2018 Published Online First 22 December 2018

\section{Check for updates}

(c) Author(s) (or their employer(s)) 2019. Re-use permitted under CC BY-NC. No commercial re-use. See rights and permissions. Published by BMJ.

To cite: Langendorf $C$ Lastrucci C, Sanou-Bicaba I, et al. Sex Transm Infect 2019:95:402-404.

\begin{abstract}
Objectives In resource-limited settings, screening pregnant women for syphilis using rapid diagnostic tests (RDTs) is a key tool in the prevention of congenital syphilis. However, most syphilis RDTs detect only treponemal antibodies (T-RDT), meaning antibiotics may be provided unnecessarily to previously treated pregnant women, particularly in non-venereal treponematoses endemic regions. We estimated the potential reduction in overtreatment when comparing T-RDT (SD Bioline) to a newer rapid test (Dual Path Platform (DPP) Screen and Confirm Assay, Chembio) detecting both treponemal and non-treponemal antibodies.
\end{abstract}

Methods Pregnant women in Déou, Burkina Faso, screened for syphilis during antenatal care (ANC) visits were prospectively enrolled in the study after providing consent. DPP and T-RDT tests were performed on whole blood specimens. Plasma was tested in an international reference laboratory by Treponema pallidum passive particle agglutination (TPPA) and quantitative rapid plasma reagin (RPR). Presumptive active syphilis was defined as a result that was both TPPA and RPR reactive. Results Of the 242 pregnant women included in the study, 91 (37.6\%) had presumptive active syphilis and $19.0 \%$ had RPR titres $\geq 8$. DPP testing did not reduce the number of pregnant women who would have been overtreated compared with T-RDT $(0.0 \%$ vs $2.5 \%$; $\mathrm{p}=0.218$ ) and had a higher proportion of underdiagnosis (48.4\% vs $2.2 \%$; $p<0.001)$. Seven women with high RPR titres $\geq 8$ would not have received treatment had only DPP testing been used.

Conclusion In the first evaluation comparing DPP with traditional screening methods in pregnant women, we saw no reduction in unnecessarily treated syphilis and an underestimation of those needing treatment. High seroprevalence in the population may indicate the presence of other treponemal infections in the area, and further study of DPP in a variety of Sahelian and other contexts is warranted.

\section{INTRODUCTION}

Screening for syphilis during antenatal care (ANC), recommended by the WHO since 2006, ${ }^{1}$ helps infected mothers start treatment early and prevents the transmission of congenital syphilis to their unborn infant. ${ }^{2}$ Caused by Treponema pallidum pallidum, syphilis is diagnosed using treponemal (T) and non-treponemal (NT) antibody testing. The former confirms the presence of $T$. pallidum antibodies. NT testing, such as rapid plasma reagin (RPR), identifies active infection. In resource-constrained settings, syphilis screening tests are usually limited to simple treponemal rapid diagnostic tests (RDTs) that allow for increased screening coverage and for same-day treatment administration.

Yet, a principal limitation of these RDTs are that they detect only $\mathrm{T}$ antibodies and that women who are $\mathrm{T}$ antibody positive due to previously treated syphilis are unnecessarily retreated. Another limitation in the syphilis diagnostic is that serological tests cannot distinguish venereal from non-venereal treponematoses: the immune response is identical. In regions where both are endemic, pregnant women may be overtreated because of the presence of $\mathrm{T}$ antibodies due to non-venereal treponematoses during their childhood. In some cases, this overtreatment also extends to the woman's partner or neonate and may inhibit women's willingness to use ANC services and to deliver in a health facility. It may also lead to social harm within her relationship or contribute to fears that a couples' child may be administered unnecessary medication.

Concerned by possible overtreatment of pregnant women, Médecins Sans Frontières (MSF) evaluated the added value of testing both T and NT antibodies in a routine ANC screening environment in Burkina Faso. We evaluated the use of the Dual Path Platform (DPP) Screen and Confirm Assay (Chembio Diagnostic Systems, USA) and compared it with reference assays. The DPP assay is a new immunochromatographic syphilis RDT that simultaneously detects T and NT antibodies and produces results in $20 \mathrm{~min}$ from capillary blood.

\section{METHODS}

The study took place at the Déou Health Center in Oudalan Province, Burkina Faso. Pregnant women presenting to ANC at the maternity ward were 
prospectively enrolled from May to August 2014. Women who received routine syphilis screening during ANC or at the time of delivery (using treponemal antibodies rapid diagnostic test (T-RDT) administered by medical staff) were eligible for inclusion. A minimum sample size of 169 true-negative participants was calculated based on McNemar's test for paired binomial data and was adjusted for an anticipated power (1- $\beta$ ) of 0.9 , a probability of type I error $(\alpha)$ of 0.05 , an assumed reduction in overtreatment of 0.5 and a true-negative prevalence of $70 \%$, resulting in a final sample size of 242 participants.

For each eligible pregnant woman who provided informed consent, DPP tests were administered by medical staff (a midwife or other auxiliary health staff) and used blood obtained through a finger prick in the ANC consultation room. T-RDT (SD Bioline Syphilis 3.0, Standard Diagnostics) and DPP testing were performed in parallel using whole blood obtained by venipuncture in the clinic's laboratory. Then, plasma was used to perform qualitative RPR (Macro-Vue, Becton Dickinson, USA) in case of a positive T-RDT result in the clinic's laboratory. As a quality control measure, a photograph of each DPP test was sent for additional interpretation by study investigators. All plasma samples, regardless of testing results at the clinical site, were sent for reference testing in an external laboratory (Institute of Tropical Medicine, Antwerp, Belgium) using reference methods: Treponema pallidum passive particle agglutination (SeroDia TP-PA, Fujirebio Diagnostics, Japan) and quantitative RPR (Macro-Vue). Medical staff, the laboratory technician and the reference laboratory were blinded to each others' results. Women with both T-RDT and qualitative RPR-positive results were treated using therapeutic protocols established by MSF and the Burkina Faso Ministry of Health. Presumptive active syphilis is serologically defined here as being both TPPA-positive and quantitative RPR-reactive at any titre. Data analysis was conducted using STATA V.12 using descriptive statistics (frequencies and proportions) for categorical variables. Means are presented with their SD and medians with their IQR. Cohen's kappa coefficient (k) was used to evaluate interuser agreement between medical staff and the laboratory technician for DPP testing. McNemar's test for paired samples was used to compare proportions of unnecessarily treated cases and incorrectly undiagnosed cases between DPP and T-RDT (significance level $\mathrm{p}=0.05$ ).

Care was provided free of charge regardless of a patient's participation in the study. All data are available on reasonable request to the corresponding author at the contact provided.

\section{RESULTS}

The 242 pregnant women assessed had a median age of 25 years (IQR 17-39). The average length of pregnancy was 27.0 weeks $( \pm 5.8)$. No women reported a history of syphilis treatment and, among these women, no symptoms of primary or secondary infection were identified by clinic midwives. Based on reference tests, 91 (37.6\%) women had presumptive active syphilis and 48 $(19.0 \%)$ had a RPR titre $\geq 8$. The vast majority of women with a reactive TPPA also had a reactive RPR $(n=91 / 101,90 \%)$.

The level of agreement between medical staff and the laboratory technician was high $(\mathrm{k}=0.95)$ for the T-line DPP tests, while agreement on the NT-line was lower $(k=0.75)$ due to difficulties interpreting weakly positive results among clinicians. Among the positive NT-lines read by the laboratory technician, 30\% (14/47) were read negative by the clinicians. Independent interpretation of DPP photos agreed with the laboratory technician's results in all cases.
Table 1 Results of T-RDT, DPP test and RPR done at the site compared with reference methods done in reference laboratory, Burkina Faso, 2014

\begin{tabular}{|c|c|c|c|c|}
\hline \multirow[t]{3}{*}{ Tests at the site } & \multicolumn{4}{|c|}{ Tests at the reference laboratory } \\
\hline & \multicolumn{4}{|l|}{ TPPA } \\
\hline & Negative & \multicolumn{2}{|l|}{ Positive } & Total \\
\hline \multicolumn{5}{|l|}{ T-RDT (clinical staff) } \\
\hline Negative & 141 & \multicolumn{2}{|l|}{6} & 147 \\
\hline Positive & 0 & \multicolumn{2}{|l|}{95} & 95 \\
\hline \multicolumn{5}{|c|}{ T-line DPP (laboratory technician) } \\
\hline Negative & 138 & \multicolumn{2}{|l|}{5} & 143 \\
\hline \multirow[t]{4}{*}{ Positive } & 3 & \multicolumn{2}{|l|}{96} & 99 \\
\hline & \multicolumn{4}{|l|}{ RPR } \\
\hline & \multirow[t]{2}{*}{ Non-reactive } & \multicolumn{2}{|l|}{ Reactive } & Total \\
\hline & & Titre $<8$ & Titre $\geq 8$ & \\
\hline \multicolumn{5}{|c|}{ Qualitative RPR* (laboratory technician) } \\
\hline Non-reactive & 6 & 23 & 0 & 29 \\
\hline Reactive & 0 & 34 & 32 & 66 \\
\hline \multicolumn{5}{|c|}{ NT-line DPP (laboratory technician) } \\
\hline Negative & 140 & 48 & 7 & 195 \\
\hline \multirow[t]{4}{*}{ Positive } & 0 & 6 & 41 & 47 \\
\hline & \multicolumn{4}{|l|}{ TPPA/RPR } \\
\hline & \multirow{2}{*}{$\begin{array}{l}\text { TPPA and/or } \\
\text { RPR negative }\end{array}$} & \multicolumn{2}{|c|}{ TPPA and RPR positive } & Total \\
\hline & & Titre $<8$ & Titre $\geq 8$ & \\
\hline \multicolumn{5}{|l|}{ T-RDT (clinical staff) } \\
\hline Negative & 145 & 2 & 0 & 147 \\
\hline Positive & 6 & 41 & 48 & 95 \\
\hline \multicolumn{5}{|c|}{ T-line+NT-line DPP (laboratory technician) } \\
\hline $\begin{array}{l}\text { T-line and/or NT-line } \\
\text { negative }\end{array}$ & 151 & 37 & 7 & 195 \\
\hline T-line and NT-line positive & 0 & 6 & 41 & 47 \\
\hline
\end{tabular}

*Tested only if T-RDT positive.

DPP, Dual Path Platform; NT, non-treponemal; RPR, rapid plasma reagin; T, treponemal; TPPA, Treponema pallidum passive particle agglutination; T-RDT, treponemal antibodies rapid diagnostic test.

Among 95 T-RDT-positive patients, the kappa agreement of qualitative RPR between the clinic's laboratory and the reference laboratory was 0.76 . All discrepancies $(n=23)$ were false negatives at the clinic's laboratory with a RPR titre $<8$ (13 with titre $=1$ ).

DPP testing conducted by the laboratory technician on whole blood did not significantly reduce the number of pregnant women who would have been overtreated (incorrectly diagnosed) compared with T-RDT testing alone $(0.0 \%$ vs $2.5 \%$, $6 / 242 ; \mathrm{p}=0.218$ ) (table 1).

Among presumptive active syphilis patients, DPP testing showed a higher proportion of pregnant women who would have been untreated (incorrectly undiagnosed) compared with T-RDT testing alone $(48.4 \%, 44 / 91$ vs $2.2 \%, 2 / 91 ; \mathrm{p}<0.001)$ (table 1). A total of $16 \%$ (7/91) of presumptive active syphilis with RPR high titres $\geq 8$ would not have been treated if they had only been screened with DPP.

T-RDT and qualitative RPR tests conducted at the clinical site resulted in $10 \%$ of cases undiagnosed overall but missed no cases among women with a RPR titre $\geq 8$ (table 1 ).

\section{DISCUSSION}

This is the first evaluation of a DPP syphilis screening assay in pregnant women in a sub-Saharan African setting where venereal and non-venereal treponematoses coexist. Though results 
did not show increased accuracy for diagnosing syphilis, they are nevertheless noteworthy; in the study area, we found that switching to a DPP-based screening algorithm would not reduce overtreatment of syphilis but would underdiagnose women who may be in need of treatment. The test's sensitivity was also lower than previous studies of behavioural high-risk groups or symptomatic patients. ${ }^{3-5}$ Despite DPP's overall usability among clinical and laboratory staff, weakly positive NT results remained more challenging for clinical staff to interpret.

Nearly $40 \%$ of this cohort was found to be both T and NT antibody positive, confirming the presence of non-venereal treponematoses (such as bejel or yaws) in the study area. ${ }^{6}$ Indeed, previous estimates in Oudalan province showed $7 \%$ of children 5-14 years of age with early stage bejel lesions, with another $41 \%$ of adults showing serological evidence of treponemal infection. ${ }^{7}$ Several of Burkina Faso's neighbouring countries also have endemic yaws. ${ }^{78}$ However, we did not expect such high proportion of young women with high level RPR titres, suggesting either incompletely treated or untreated bejel during childhood or current asymptomatic active syphilis infection. Exploring baseline T/NT antibody seroprevalence in any population will remain important to identify the most efficient strategy to screen and treat treponemal diseases in such complex settings. There is a need to determine the level of endemicity of non-venereal treponematoses in the region by testing children and non-pregnant adults. There is an additional need to potentially control the disease through the use of mass treatment with single-dose oral azithromycin. ${ }^{9}$ Finally, increasing the specificity of serological syphilis screening among pregnant women may lead to medication being prioritised for those who most need it, particularly in countries affected by increasingly common shortages of benzathine penicillin.

Ultimately, innovative disease screening tools should not replace established methods until their added value is confirmed in context-specific evaluations. In the study setting in Burkina Faso, despite excellent assay performance in detecting $\mathrm{T}$ antibodies, DPP showed low sensitivity for NT detection. The lower sensitivity was also observed at higher RPR titres, increasing the risk of congenital syphilis. ${ }^{10}$ Previously, the test performed well in people diagnosed with clinical syphilis or those at risk of STIs, especially when patients' RPR titres were high $(\geq 8),{ }^{3-5}$ suggesting that test performance may vary with cohort composition and that, in this context, a unique T-RDT remains the most accurate and cost-effective choice $(<€ 1$ per test) for pregnant women. More expensive DPP testing (€2-3 per test) should be re-evaluated in other environments and populations to establish whether, in those contexts, combined T/NT antibody detection will significantly improve the prevention of congenital syphilis transmission, benzathine penicillin use or overall patient care.

Handling editor Catherine A Ison
Acknowledgements We are grateful to the pregnant women who participated in this study and also wish to thank the Ministry of Health in Burkina Faso for their support. We are also indebted to the clinical and laboratory staff in Burkina Faso and in Belgium who made this study happen. We are thankful to Janet Ousley for her valuable support.

Contributors Céline Langendorf, Céline Lastrucci, KB and TC conceived of and designed the work; Céline Langendorf, Céline Lastrucci, KB, M-HK and IS-B were responsible for the acquisition, analysis and interpretation of the data; Céline Langendorf and Céline Lastrucci prepared the manuscript, and TC revised the work critically for important intellectual content and provided final approval of the version to be published. All authors agree to be accountable for all aspects of the work.

Funding Médecins Sans Frontières funded this study in its entirety. Epicentre receives core funding from Médecins Sans Frontières, and no pharmaceutical or other external grants were received during the development or implementation of this study.

Competing interests None declared.

Patient consent for publication Not required.

Ethics approval The study was approved by the Comite de Protection des Personnes (Saint Germain-en-Laye, France; approval number 14019), the Comité d'Ethique du Ministère de la Santé Publique in Burkina Faso (2014-3-021) and the Institutional Review Board of the ITM (Belgium, approval number 935/14).

Provenance and peer review Not commissioned; externally peer reviewed.

Data sharing statement All data are available upon reasonable request to the corresponding author at the contact provided.

Open access This is an open access article distributed in accordance with the Creative Commons Attribution Non Commercial (CC BY-NC 4.0) license, which permits others to distribute, remix, adapt, build upon this work non-commercially, and license their derivative works on different terms, provided the original work is properly cited, appropriate credit is given, any changes made indicated, and the use is non-commercial. See: http://creativecommons.org/licenses/by-nc/4.0/

\section{REFERENCES}

1 World Health Organisation. WHO guideline on syphilis screening and treatment for pregnant women. 2017. Available: http://apps.who.int/iris/bitstream/10665/259003/ 1/9789241550093-eng.pdf?ua=1

2 Gomez GB, Kamb ML, Newman LM, et al. Untreated maternal syphilis and adverse outcomes of pregnancy: a systematic review and meta-analysis. Bull World Health Organ 2013;91:217-26.

3 Castro AR, Esfandiari J, Kumar S, et al. Novel point-of-care test for simultaneous detection of nontreponemal and treponemal antibodies in patients with syphilis. J Clin Microbiol 2010;48:4615-9.

4 Causer LM, Kaldor JM, Conway DP, et al. An evaluation of a novel dual treponemal/ nontreponemal point-of-care test for syphilis as a tool to distinguish active from past treated infection. Clin Infect Dis 2015;61:184-91.

5 Yin YP, Chen XS, Wei WH, et al. A dual point-of-care test shows good performance in simultaneously detecting nontreponemal and treponemal antibodies in patients with syphilis: a multisite evaluation study in China. Clin Infect Dis 2013;56:659-65.

6 Kirakoya-Samadoulougou F, Defer MC, Yaro S, et al. Low seroprevalence of syphilis in Burkina Faso. Sex Transm Infect 2011;87:35-7.

7 Gazin P, Meynard D. [A clinical and serologic survey of bejel in north Burkina Faso]. Bull Soc Pathol Exot Filiales 1988;81:827-31.

8 Kazura JW. Yaws eradication--a goal finally within reach. $N$ Engl J Med 2015;372:693-5.

9 Mitjà 0 , Houinei W, Moses $P$, et al. Mass treatment with single-dose azithromycin for yaws. N Engl J Med 2015;372:703-10.

10 Watson-Jones D, Changalucha J, Gumodoka B, et al. Syphilis in pregnancy in Tanzania. I. Impact of maternal syphilis on outcome of pregnancy. J Infect Dis 2002;186:940-7. 